
\title{
28 Research Square \\ Clinical Characteristics, Treatment Outcomes, And Genetic Alterations of Radiation-Induced Gliomas
}

Makoto Ohno ( $\nabla$ mohno@ncc.go.jp )

National Cancer Center Hospital https://orcid.org/0000-0001-8031-4306

Yasuji Miyakita

National Cancer Center Hospital

Masamichi Takahashi

National Cancer Center Hospital

Shunsuke Yanagisawa

National Cancer Center Hospital

Yukie Tamura

National Cancer Center Hospital

Daisuke Kawauchi

National Cancer Center Hospital

Miyu Kikuchi

National Cancer Center Hospital

Hiroshi Igaki

National Cancer Center Hospital

Akihiko Yoshida

National Cancer Center Hospital

Kaishi Satomi

National Cancer Center Hospital

Yuko Matsushita

National Cancer Center Hospital

Koichi Ichimura

National Cancer Center Research Institute

Yoshitaka Narita

National Cancer Center Hospital

\section{Research Article}

Keywords: Radiation-induced glioma, IDH1/2 mutations, Secondary neoplasms, long-term survivors of childhood malignancies, Reirradiation

Posted Date: November 15th, 2021 
DOI: https://doi.org/10.21203/rs.3.rs-1057891/v1

License: (c) (1) This work is licensed under a Creative Commons Attribution 4.0 International License. Read Full License 


\section{Abstract}

Purpose: We aimed to elucidate the clinical characteristics, treatment outcomes, and genetic alterations in patients with radiation-induced glioma (RIG).

Methods: Patients with high-grade glioma who satisfied the Cahan's criteria for RIG in our database during 2001-2021 were included. Isocitrate dehydrogenase (IDH) 1 and 2, telomerase reverse transcriptase (TERT) promoter, B-Raf (BRAF), and histone H3.3 (H3F3A) and methylation status of the 06-methylguanine DNA methyltransferase (MGMT) promoter were analyzed.

Methods: We identified 11 patients with RIG, including 7 glioblastoma (GBM), IDH1/2-wildtype; 2 GBM, not otherwise specified (NOS); 1 anaplastic astrocytoma (AA), IDH1/2-wildtype; and 1 AA, NOS. The median latency period was 17 years (range: 9-30 years). The median progression-free survival (PFS) and median survival time (MST) were 11.3 months and 28.3 months, respectively. The median PFS in patients treated with initial reirradiation $(\mathrm{N}=5)$ tended to be longer than that in patients without initial reirradiation $(\mathrm{N}=6)$ (17.0 vs 8.1 months; $\mathrm{p}=0.51$ ), but not MST (29.6 vs 27.4 months; $\mathrm{p}=0.28$ ). There were no alterations in IDH1/2, TERT promoter, BRAF and H3F3A mutations. Two tumors had a hypermethylated MGMT promoter. In one case, different IDH2 mutation status between primary and secondary tumor was useful to diagnose the secondary tumor as RIG.

Conclusions: RIG may occur more than 20 years after the treatment for primary disease; therefore, longterm follow-up is needed. Reirradiation could be a therapeutic option for RIG. Identifying IDH1/2 mutation status has a diagnostic impact on establishing RIG in cases of recurrent glioma.

\section{Introduction:}

Radiotherapy has been used for cancer treatment, including pediatric brain tumors or hematological malignancies, such as glioma, medulloblastoma, germ cell tumors, and leukemia. Despite of the overall improvement in the survival rates of patients with these tumors, patients treated with radiotherapy are at risk of long-term neurological complications such as the development of progressive leukoencephalopathy, arteritis, hypopituitarism, and hypothalamic insufficiency.[1] One of the most serious late consequences of radiotherapy is secondary neoplasms, which occurs in rare cases but represents a major cause of mortality in long-term survivors of childhood malignancies.[2-6] Among radiation-induced brain tumors, meningiomas and gliomas are the most frequently reported secondary neoplasms.[1] The cumulative risk of occurrence of secondary brain tumors after radiation therapy for pituitary adenomas is $2.0 \%$ at 10 years and $2.4 \%$ at 20 years, which is 10.5 times higher than that seen in the general population.[3] The cumulative risk of occurrence of secondary brain tumors among long-term survivors of acute lymphoblastic leukemia is $0.8 \%$ at 10 years and $1.87 \%$ at 20 years.[2]

Radiation-induced gliomas (RIGs) are typically high-grade tumors. Their treatment is usually challenging and the clinical outcome is generally poor.[3,5-7] The median overall survival (OS) for patients with RIG was 11 months, with a 2-year survival rate of 20.2\%.[6] Although several studies or review articles have 
proposed combination therapy with radiation and chemotherapy is a potential treatment option, the optimal approach for managing and treating RIG has not well defined.[6] Moreover, there have been few reports that investigated genetic alterations of RIG.[8-14]

In this study, we investigated the clinical characteristics, treatment outcomes, and genetic alterations of 11 patients with RIG with the aim to identify the optimal follow-up period from the treatment of primary disease, therapeutic strategy, molecular characteristics and their clinical impact of RIG.

\section{Methods And Materials:}

\section{Patient characteristics}

This study was conducted as a retrospective observational study. We reviewed our department database between 2001-2021. We included patients who satisfied the Cahan's criteria, which were as follows: (1) the tumor must originate in the previously irradiated region (but not necessarily in the full-dose region), (2) there must be a sufficient latency time from irradiation to the onset of the postradiation tumor; (3) the tumor histology must be different from that of the primary one, and (4) the patient must not have pathologies favoring development of tumors: Li-Fraumeni's disease, von Recklinghausen's disease, tuberous sclerosis, xeroderma pigmentation, and retinoblastoma.[6, 15, 16]

Patients' clinical, operative, and radiological information was reviewed, and data of the following variables were collected: clinical and treatment history before RIG diagnosis, clinical and treatment history after RIG diagnosis, date of operation of RIG, postoperative therapy of RIG, date of tumor recurrence of RIG, date of death or last hospital visit, extent of resection of RIG, and treatment after tumor recurrence of RIG. The extent of resection of RIG was determined based on the surgeon's operative notes and postoperative imaging studies, classified as follows: total, if $100 \%$ of the contrast-enhanced lesion was resected; subtotal, if $95 \%-99 \%$ of the lesion was resected; partial, if $<94 \%$ of the lesion was resected, or as a biopsy.[17] All patients were re-diagnosed by neuropathologists at our hospital according to the World Health Organization 2016 classification.[18]

\section{Molecular analysis}

Tumor DNA was extracted from frozen tumor tissues of all cases using a DNeasy Blood \& Tissue Kit (Qiagen, Tokyo, Japan). The presence of hotspot mutations in isocitrate dehydrogenase (IDH) 1 (R132) and IDH2 (R172) was assessed by pyrosequencing, as described previously.[19] Pyrosequencing assays were designed to detect all known mutations in these codons.[19] The two mutation hotspots in the telomerase reverse transcriptase (TERT) promoter were analyzed in all tumors by Sanger sequencing and/or pyrosequencing, as reported previously.[20] The mutation hotspots at codons 27 and 34 of histone H3.3 (H3F3A) and those at codon 600 of B-Raf (BRAF) were analyzed by Sanger sequencing and/or pyrosequencing.[21] The methylation status of the 0-6-methylguanine DNA methyltransferase (MGMT) promoter was analyzed using bisulfite modification of the tumor genomic DNA, followed by 
pyrosequencing, as previously described.[20] Methylation was considered positive when its mean level at the $16 \mathrm{CpG}$ site was greater than $16 \%[17,20]$

\section{Statistical analysis}

The latent period was defined as the interval between the date of diagnosis of the primary disease and that of RIG. OS was defined as the interval between the date of RIG surgery and that of death or the last follow-up, whichever occurred first. Progression-free survival (PFS) was defined as the interval between the date of RIG surgery and that of the detection of progression, death, or last follow-up. These times were calculated using the Kaplan-Meier method and compared using the log-rank test. Statistical significance was set at $p<0.05$. Statistical tests were performed using JMP® software, ver. 15.1.0 for Mac (SAS Institute Japan, Tokyo, Japan), and GraphPad Prism®, ver. 9.0 (GraphPad Software, La Jolla, CA, USA).

\section{Results:}

\section{Patient characteristics of primary disease}

We identified 11 patients who satisfied the Cahan's criteria and had RIG.[6, 15, 16] Patient characteristics of the primary disease are summarized in Table 1 . The median age of the 11 patients was 12 years (range: 1-39 years), and there was no sex predominance (male: 6, female: 5). The primary diseases included germinomas $(n=2)$, acute lymphoblastic leukemias $(n=2)$, medulloblastomas $(n=3)$, diffuse astrocytoma (DA; $n=1), I D H 2$-mutant, pilocytic astrocytoma $(n=1)$, pituitary adenoma $(n=1)$, and metastatic brain tumor from lung cancer $(n=1)$. All patients received cranial radiation (range: $12-101.5$ Gy). The median latent time between primary disease and RIG was 17 years (range: $9-30$ years) (Table 1).

\section{Patient characteristics and treatment of RIGs}

The characteristics of patients with RIG are summarized in Table 2. The median age of the 11 patients was 34 years (range: 10-49 years). Seven patients had glioblastoma (GBM), IDH1/2-wildtype; 2 had GBM, not otherwise specified (NOS); 1 had anaplastic astrocytoma (AA), IDH1/2-wildtype; and 1 had AA, NOS. All patients underwent tumor removal or biopsy and were diagnosed based on histopathological examination. Four patients had comorbidities, which were related to primary radiation therapy: two had hypopituitarism, one had decreased visual acuity, and one had mental retardation. On preoperative magnetic resonance imaging (MRI), all tumors were shown as gadolinium contrast-enhanced diseases (Fig. 1A-K) and five of them were cystic (Fig. 1C, E, G, H, and J). Two patients had multiple intraparenchymal lesions (Fig. $1 \mathrm{C}$ and F), and one had a right cerebellar lesion with cerebrospinal dissemination (Fig. 1D).

For postoperative treatment, six patients were treated with chemotherapy and five received reirradiation (ReRT) combined with chemotherapy. The chemotherapy regimen included temozolomide (TMZ; n=6), 
nimustine hydrochloride ( $\mathrm{ACNU} ; \mathrm{n}=2)$, TMZ and bevacizumab (TMZ/Bev; $\mathrm{n}=2$ ), and carboplatin and etoposide $(n=1)$. The ReRT regimens were as follows: 40 Gy in 15 fractions, 40 Gy in 20 fractions, 50 Gy in 25 fractions, 60 Gy in 30 fractions, and 66 Gy in 33 fractions.

Ten patients had tumor recurrences, and seven patients received further treatments for recurrent tumors. All the patients were treated with chemotherapy. Three patients who had not undergone ReRT during the initial treatment of RIG received ReRT at the time of recurrence. Two patients with supratentorial tumors had tumor recurrence in the cerebellum at a distance from the initial location and received stereotactic radiotherapy consisting of $42 \mathrm{~Gy}$ in $7 \mathrm{Fr}$ (Table 2).

\section{Treatment outcomes of RIGs}

The median PFS and median survival time (MST) in patients with RIG were 11.3 months and 28.3 months, respectively (Fig. 2A and 2B, respectively). The median PFS in patients initially treated with ReRT $(\mathrm{N}=5)$ was 17.0 months, which tended to be longer than that in patients without ReRT $(\mathrm{N}=6)$, with 8.1 months, although the difference was not significant $(p=0.51)$ (Fig. 2C). The MST in patients with ReRT $(\mathrm{N}=5)$ and those without ReRT $(\mathrm{N}=6)$ were 29.6 and 27.4 months, respectively, without any significant difference $(p=0.28)$ (Fig. 2D). None of the patients developed symptomatic radiation necrosis, which might be related to a high cumulative radiation dose.

\section{Genetic alterations of RIGs}

The genetic alterations of eight patients whose tumor samples were available for analysis are summarized in Table 3. There were no alterations in the IDH1/2 and TERT promoters in eight cases and $B R A F$ and H3F3A mutations in six cases. Two tumors had hypermethylated MGMT promoter status, whereas the other six had hypomethylated status.

\section{Case presentation}

A 12-year-old female patient (Case 9) initially presented with a cataplectic attack, and 3 years later, MRI revealed a left frontal non-contrast-enhanced tumor (Fig. 3A). She underwent subtotal resection and was diagnosed with DA. After the operation, she received radiation therapy at a dose of 60 Gy in 30 fractions and chemotherapy with ACNU. Seventeen years after treatment for DA, she developed tumor recurrence just posterior to the primary tumor (Fig. 1I) and underwent biopsy. The tumor was diagnosed as GBM, and the IDH1/2 gene was wildtype (Fig. 3B). We also performed pyrosequencing analysis of the IDH1/2 gene in the primary tumor and found that the tumor had an IDH2 mutation (Fig. 3C). Based on the difference in IDH1/2 mutation status, we thought that the current GBM, IDH1/2-wildtype, did not develop from the primary DA, IDH2-mutant, but was likely to be a de novo tumor that was related to the previous radiation therapy. Therefore, we diagnosed the GBM, IDH1/2-wildtype, as RIG. The patient received radiation therapy at a dose of 60 Gy in 30 fractions combined with TMZ; however, the patient died 35.1 months after the diagnosis of GBM, IDH1/2-wildtype. 


\section{Discussion:}

In this study, we observed a median latent time of 17 years with ranging from 9 to 30 years, suggesting the importance of a much long follow-up period. We performed ReRT for five patients at initial treatment of RIG and for three patients at the time of recurrence in 11 patients with RIG and found that the median PFS and MST were 11.3 months and 28.3 months, respectively. We identified no genetic alterations in IDH1/2 and TERT promoters or in H3F3A, and BRAF genes, which are commonly observed in spontaneous high-grade gliomas. In addition, we found that the evaluation of IDH1/2 mutation status was helpful in establishing the diagnosis of RIG in cases whose $I D H 1 / 2$ mutation status were different between primary and secondary glioma.

In our study, the median age of patients with primary disease was 13.5 years, and 7 of 10 patients were younger than 20 years, which was comparable to the data reported in previous studies.[5,6] Our primary diseases included ALL, meduloblastoma, germinoma, pituitary adenoma, PA, DA, and metastatic brain tumors, which are common primary diseases. Yamanaka et al. reviewed 296 RIGs in the literature and reported that the primary tumors included hematological malignancy in $35.1 \%$ of the patients, medulloblastoma in $12.8 \%$, pituitary adenoma in $11.8 \%$, low-grade glioma in $9.4 \%$, craniopharyngioma in $6.4 \%$, germ cell tumor in $4.3 \%$, and metastatic brain tumor in $1.6 \%$ of the patients.[6]

Our cohort included one patient with metastatic brain tumor from lung adenocarcinoma harboring epidermal growth factor mutations. The patient developed RIG 10 years after whole-brain radiotherapy for multiple lesions and died due to RIG (Case 10). Based on clinical and radiological findings (Fig. 1J), we assumed that the cystic lesion developing in the right frontal lobe was a new recurrence of metastatic brain tumor; however, the histological diagnosis was glioblastoma. This case indicates the possible risk of developing RIG after radiotherapy for metastatic brain tumor and emphasizes the importance of histopathological confirmation, especially in cases where tumor recurrence after radiotherapy has occurred after a long time. Although the incidence of RIG with the primary disease of metastatic brain tumor is rare,[6,22] it is important for clinicians to be aware that the advances in cancer therapy and the increased use of radiotherapy for metastatic brain tumors might allow patients to develop RIG.[22]

Our median latent time from the primary cancer treatment to the development of RIG was 17 years and varied from 9 to 30 years in our cohort. Previous reports showed that the median latency period was 911 years, and most RIGs occurred within 15 years. [4, 5] However, in our series, four out of 11 patients $(36.4 \%)$ had a latent period of 20 years or more. Nakao et al. also reported that the latency period was more than 20 years in four patients.[12] These results indicate that pediatric patients with primary diseases successfully treated with radiation therapy have a risk of developing secondary neoplasm more than 20 years after the initial treatment. From the clinical point of view, these patients should be carefully monitored much longer than we have anticipated. Based on our results, we conclude that the follow-up time must be extended to 30 years.

One of the controversies in the treatment of RIG is whether ReRT may have a therapeutic effect on tumors that were induced by previous radiation. In our study, five patients underwent ReRT during the initial 
treatment of RIG, and three had ReRT at the time of RIG recurrence. The median PFS and MST were 11.3 and 28.3 months, respectively, which were better than those reported in previous studies.[5, 6, 23] Moreover, we found that patients treated with initial ReRT tended to have a longer PFS than those without initial ReRT, but MST was similar between these groups. The crossover of ReRT at the time of recurrence might prolong the OS in patients who have not undergone initial ReRT. This is a key finding, as it emphasizes the importance of ReRT in the treatment of RIG, regardless of the timing. Paulino et al. reported that patients who underwent ReRT for RIG showed better survival rates than those who did not (13 vs. 8 months; $p=0.0009$ ), suggesting the efficacy of ReRT in treating these tumors.[5] Yamanaka et al. also reported that MST in patients who received surgery, chemotherapy, and ReRT was 18 months, whereas that in those who did not undergo chemotherapy was 9 months $(p=0.0006)$, indicating that intensive combination of ReRT and chemotherapy is a potential treatment option for RIG.[6] Recent molecular analysis suggested that RIGs harbor a largely homogeneous genetic and epigenetic profile of sporadic pediatric receptor tyrosine kinase I GBM, which could be particularly vulnerable to ionizing radiation. $[13,14]$ These clinical and molecular evidences support the use of ReRT in the treatment of RIG. Further studies are needed to confirm the efficacy of ReRT in a large cohort.

Another concern in the treatment of RIG is ReRT-related radiation necrosis. We did not observe symptomatic radiation necrosis in our patients. According to literature reviews, the risk of developing necrosis was less than $10 \%$ in the patients who underwent ReRT treatment for RIG.[5] The interval between the first and second radiation sessions is usually more than 10 years and most patients with RIG die within three years, therefore, the late complication related to ReRT might not be clinically relevant. In addition, two patients (Cases 10 and 11) in our cohort received Bev combined with postoperative ReRT in the treatment of RIG, and did not develop symptomatic radiation necrosis during the follow-up period. These cases suggest that the use of Bev combined with ReRT may be a therapeutic option to minimize radiation-related risk. It will be of interest to investigate the efficacy of the combination therapy of ReRT and Bev, or to develop the optimal dose-fractionation schedule of ReRT with Bev, which meets efficacy and safety in the treatment of RIG.

We investigated genetic alterations in eight patients whose tumor samples were available. We found no alterations in the IDH1/2 and TERT promoter or in H3F3A and BRAF, which are commonly observed in spontaneous high-grade gliomas. Two patients had a hypermethylated MGMT promoter, while the other 6 patients had a hypomethylated MGMT promoter. These results are consistent with those of previous reports.[9-14] A genetic profiling study on 12 RIGs found that all tumors lacked alterations in $I D H 1 / 2$, TERT promoter, H3F3A, chromosome 7 gain, and chromosome 10 loss. In addition, the study reported that radiation-induced high-grade gliomas demonstrated significantly increased intrachromosomal copy number breakpoints, likely because of DNA double-strand breaks induced by previous therapeutic radiation.[11] Recently, RIG was shown to be characterized by platelet-derived growth factor receptor alpha (PDGFRA) amplification, loss of cyclin-dependent kinase inhibitor $2 \mathrm{~A}$ and $\mathrm{B}(C D K N 2 A / B)$, and absence of histone 3 or $I D H 1 / 2$ mutations, suggesting the molecularly distinct gliomagenesis from spontaneous high-grade gliomas.[13] Moreover, drug screening study suggested microtubule inhibitors/stabilizers, DNA-damaging agents, MEK inhibitors, and proteasome inhibitors as potentially 
effective therapies.[14] The understanding of the underlying biology will make it possible to provide new therapeutic approaches in the treatment of RIG.

In Case 9, the difference in $I D H 1 / 2$ mutation status between the primary and secondary tumors was helpful in establishing the diagnosis of RIG. This case highlights the importance of identifying the IDH1/2 mutation status in both primary and secondary gliomas. In addition, it also reminded us of the potential risk of developing RIG after radiation therapy for diffuse gliomas and the importance of long-term followup. Prior studies consistently report that RIG does not harbor IDH1/2 mutations[11-13], therefore, identifying the wild-type $I D H 1 / 2$ is diagnostic relevant. We recommend evaluating $I D H 1 / 2$ mutation status between the primary and secondary gliomas, especially in cases with recurrent glioma occurring long time after successful initial treatment.

Our study had certain limitations. First, this was a retrospective study, and we did not investigate the molecular alterations in three patients because tissue samples were unavailable. Further studies are needed to elucidate the molecular characteristics of RIG. Second, our cohort was too small to draw definitive conclusions. We acknowledge that the power of survival analysis regarding the usefulness of ReRT and the ReRT-related toxicity was limited by the sample size; therefore, our results need to be confirmed in larger cohort studies.

\section{Conclusions:}

RIG may occur more than 20 years after successful treatment for primary disease using radiotherapy, thus, the follow-up time must be extended to 30 years. ReRT can be considered as a therapeutic option for the treatment of RIG, and ReRT combined with Bev or optimal dose-fractionation schedule with Bev needs to be investigated. Identifying the $I D H 1 / 2$ mutation status has a diagnostic impact on establishing RIG in recurrent glioma.

\section{Declarations:}

\section{Statements and Declarations}

Author contributions: M.O. and Y.N. designed the study. M.O., Y.M., M.T., S.Y., Y.T., D.K, M.K, H.I, and Y.N. contributed to patients' management and treatment. A.Y. and K.S. contributed to diagnosis. M.O., Y.M., M.T., S.Y., Y.T., Y.M., K.I. and Y.N. contributed to sample collection, molecular analysis, data acquisition and interpretation. M.O. conducted the statistical analysis. M.O. and Y.N wrote the manuscript, and all the authors reviewed, edited and approved this manuscript.

Funding: None

Acknowledgments: A part of this study was presented at the 19th International Symposium on Pediatric Neuro-Oncology meeting. 
Data Availability Statement for this Work: The datasets used and/or analyzed during the current study are available from the corresponding author on reasonable request.

Conflict of Interest: H. Igaki received grant from HekaBio, Elekta KK, and CICS, consulting fee from HekaBio, and lecture fee from Varian, Itochu, and CICS. All authors report no disclosures relevant to the manuscript.

Ethical considerations: All procedures performed in this study were in accordance with the ethical standards of the institutional review board and the 1964 Helsinki Declaration and its later amendments. This study was approved by the Institutional Review Board of National Cancer Center (2004-066 or 2007086). Written informed consent was obtained from all participants.

\section{References:}

1. Carr CM, Benson JC, DeLone DR, Diehn FE, Kim DK, Merrell KW, Nagelschneider AA, Madhavan AA, Johnson DR (2021) Intracranial long-term complications of radiation therapy: an image-based review. Neuroradiology 63: 471-482 doi:10.1007/s00234-020-02621-7

2. Hijiya N, Hudson MM, Lensing S, Zacher M, Onciu M, Behm FG, Razzouk BI, Ribeiro RC, Rubnitz JE, Sandlund JT, Rivera GK, Evans WE, Relling MV, Pui CH (2007) Cumulative incidence of secondary neoplasms as a first event after childhood acute lymphoblastic leukemia. JAMA 297: 1207-1215 doi:10.1001/jama.297.11.1207

3. Minniti G, Traish D, Ashley S, Gonsalves A, Brada M (2005) Risk of second brain tumor after conservative surgery and radiotherapy for pituitary adenoma: update after an additional 10 years. J Clin Endocrinol Metab 90: 800-804 doi:10.1210/jc.2004-1152

4. Neglia JP, Friedman DL, Yasui Y, Mertens AC, Hammond S, Stovall M, Donaldson SS, Meadows AT, Robison LL (2001) Second malignant neoplasms in five-year survivors of childhood cancer: childhood cancer survivor study. J Natl Cancer Inst 93: 618-629 doi:10.1093/jnci/93.8.618

5. Paulino AC, Mai WY, Chintagumpala M, Taher A, Teh BS (2008) Radiation-induced malignant gliomas: is there a role for reirradiation? Int J Radiat Oncol Biol Phys 71: 1381-1387

doi:10.1016/j.ijrobp.2007.12.018

6. Yamanaka R, Hayano A, Kanayama T (2018) Radiation-induced gliomas: a comprehensive review and meta-analysis. Neurosurg Rev 41: 719-731 doi:10.1007/s10143-016-0786-8

7. Pettorini BL, Park YS, Caldarelli M, Massimi L, Tamburrini G, Di Rocco C (2008) Radiation-induced brain tumours after central nervous system irradiation in childhood: a review. Childs Nerv Syst 24: 793805 doi:10.1007/s00381-008-0631-7 
8. Hiraki T, Fukuoka K, Mori M, Arakawa Y, Matsushita Y, Hibiya Y, Honda S, Kobayashi M, Tanami Y, Ichimura K, Hirato J, Kurihara J, Nakazawa A, Koh K (2021) Application of Genome-Wide DNA Methylation Analysis to Differentiate a Case of Radiation-Induced Glioblastoma From Late-Relapsed Medulloblastoma. J Neuropathol Exp Neurol 80: 552-557 doi:10.1093/jnen/nlab043

9. Izycka-Swieszewska E, Bien E, Stefanowicz J, Szurowska E, Szutowicz-Zielinska E, Koczkowska M, Sigorski D, Kloc W, Rogowski W, Adamkiewicz-Drozynska E (2018) Malignant Gliomas as Second Neoplasms in Pediatric Cancer Survivors: Neuropathological Study. Biomed Res Int 2018: 4596812 doi:10.1155/2018/4596812

10. Kajitani T, Kanamori M, Saito R, Watanabe Y, Suzuki H, Watanabe M, Kure S, Tominaga T (2018) Three case reports of radiation-induced glioblastoma after complete remission of acute lymphoblastic leukemia. Brain Tumor Pathol 35: 114-122 doi:10.1007/s10014-018-0316-1

11. Lopez GY, Van Ziffle J, Onodera C, Grenert JP, Yeh I, Bastian BC, Clarke J, Oberheim Bush NA, Taylor J, Chang S, Butowski N, Banerjee A, Mueller S, Kline C, Torkildson J, Samuel D, Siongco A, Raffel C, Gupta N, Kunwar S, Mummaneni P, Aghi M, Theodosopoulos P, Berger M, Phillips JJ, Pekmezci M, Tihan T, Bollen AW, Perry A, Solomon DA (2019) The genetic landscape of gliomas arising after therapeutic radiation. Acta Neuropathol 137: 139-150 doi:10.1007/s00401-018-1906-z

12. Nakao T, Sasagawa Y, Nobusawa S, Takabatake Y, Sabit H, Kinoshita M, Miyashita K, Hayashi Y, Yokoo H, Nakada M (2017) Radiation-induced gliomas: a report of four cases and analysis of molecular biomarkers. Brain Tumor Pathol 34: 149-154 doi:10.1007/s10014-017-0292-x

13. Deng MY, Sturm D, Pfaff E, Sill M, Stichel D, Balasubramanian GP, Tippelt S, Kramm C, Donson AM, Green AL, Jones C, Schittenhelm J, Ebinger M, Schuhmann MU, Jones BC, van Tilburg CM, Wittmann A, Golanov A, Ryzhova M, Ecker J, Milde T, Witt O, Sahm F, Reuss D, Sumerauer D, Zamecnik J, Korshunov A, von Deimling A, Pfister SM, Jones DTW (2021) Radiation-induced gliomas represent H3-/IDH-wild type pediatric gliomas with recurrent PDGFRA amplification and loss of CDKN2A/B. Nat Commun 12: 5530 doi:10.1038/s41467-021-25708-y

14. DeSisto J, Lucas JT, Jr., Xu K, Donson A, Lin T, Sanford B, Wu G, Tran QT, Hedges D, Hsu CY, Armstrong GT, Arnold M, Bhatia S, Flannery P, Lemma R, Hardie L, Schuller U, Venkataraman S, Hoffman LM, Dorris K, Mulcahy Levy JM, Hankinson TC, Handler M, Liu AK, Foreman N, Vibhakar R, Jones K, Allen S, Zhang J, Baker SJ, Merchant TE, Orr BA, Green AL (2021) Comprehensive molecular characterization of pediatric radiation-induced high-grade glioma. Nat Commun 12: 5531 doi:10.1038/s41467-021-25709-x

15. Cahan WG (1998) Radiation-induced sarcoma--50 years later. Cancer 82: 6-7 doi:10.1002/(sici)10970142(19980101)82:1<6::aid-cncr2>3.0.co;2-t

16. Cahan WG, Woodard HQ, et al. (1948) Sarcoma arising in irradiated bone; report of 11 cases. Cancer 1: 3-29 doi:10.1002/1097-0142(194805)1:1<3::aid-cncr2820010103>3.0.co;2-7 
17. Ohno M, Miyakita Y, Takahashi M, Igaki H, Matsushita $Y$, Ichimura K, Narita Y (2019) Survival benefits of hypofractionated radiotherapy combined with temozolomide or temozolomide plus bevacizumab in elderly patients with glioblastoma aged $>/=75$ years. Radiat Oncol 14: 200 doi:10.1186/s13014-0191389-7

18. Louis DN, Ohgaki H, Wiestler OD, WK C (2016) WHO classification of tumours of the central nervous system. Revise 4th edition. . Lyon: IARC Press

19. Arita H, Narita Y, Matsushita Y, Fukushima S, Yoshida A, Takami H, Miyakita Y, Ohno M, Shibui S, Ichimura K (2015) Development of a robust and sensitive pyrosequencing assay for the detection of IDH1/2 mutations in gliomas. Brain Tumor Pathol 32: 22-30 doi:10.1007/s10014-014-0186-0

20. Arita H, Narita Y, Fukushima S, Tateishi K, Matsushita Y, Yoshida A, Miyakita Y, Ohno M, Collins VP, Kawahara N, Shibui S, Ichimura K (2013) Upregulating mutations in the TERT promoter commonly occur in adult malignant gliomas and are strongly associated with total 1p19q loss. Acta Neuropathol 126: 267276 doi:10.1007/s00401-013-1141-6

21. Arita H, Yamasaki K, Matsushita Y, Nakamura T, Shimokawa A, Takami H, Tanaka S, Mukasa A, Shirahata M, Shimizu S, Suzuki K, Saito K, Kobayashi K, Higuchi F, Uzuka T, Otani R, Tamura K, Sumita K, Ohno M, Miyakita Y, Kagawa N, Hashimoto N, Hatae R, Yoshimoto K, Shinojima N, Nakamura H, Kanemura Y, Okita Y, Kinoshita M, Ishibashi K, Shofuda T, Kodama Y, Mori K, Tomogane Y, Fukai J, Fujita K, Terakawa Y, Tsuyuguchi N, Moriuchi S, Nonaka M, Suzuki H, Shibuya M, Maehara T, Saito N, Nagane M, Kawahara N, Ueki K, Yoshimine T, Miyaoka E, Nishikawa R, Komori T, Narita Y, Ichimura K (2016) A combination of TERT promoter mutation and MGMT methylation status predicts clinically relevant subgroups of newly diagnosed glioblastomas. Acta Neuropathol Commun 4: 79 doi:10.1186/s40478016-0351-2

22. Nukaga S, Naoki K, Yasuda H, Kawada I, Ohara K, Soejima K, Betsuyaku T (2018) Secondary Brain Neoplasm after Stereotactic Radiosurgery in Patients with Metastatic Non-small Cell Lung Cancer. Intern Med 57: 2383-2387 doi:10.2169/internalmedicine.0184-17

23. Salvati M, D'Elia A, Melone GA, Brogna C, Frati A, Raco A, Delfini R (2008) Radio-induced gliomas: 20year experience and critical review of the pathology. J Neurooncol 89: 169-177 doi:10.1007/s11060-008$9565-x$

\section{Tables:}

Due to technical limitations, tables 1,2 and 3 are only available as a download in the Supplemental Files section.

\section{Figures}




\section{Figure 1}
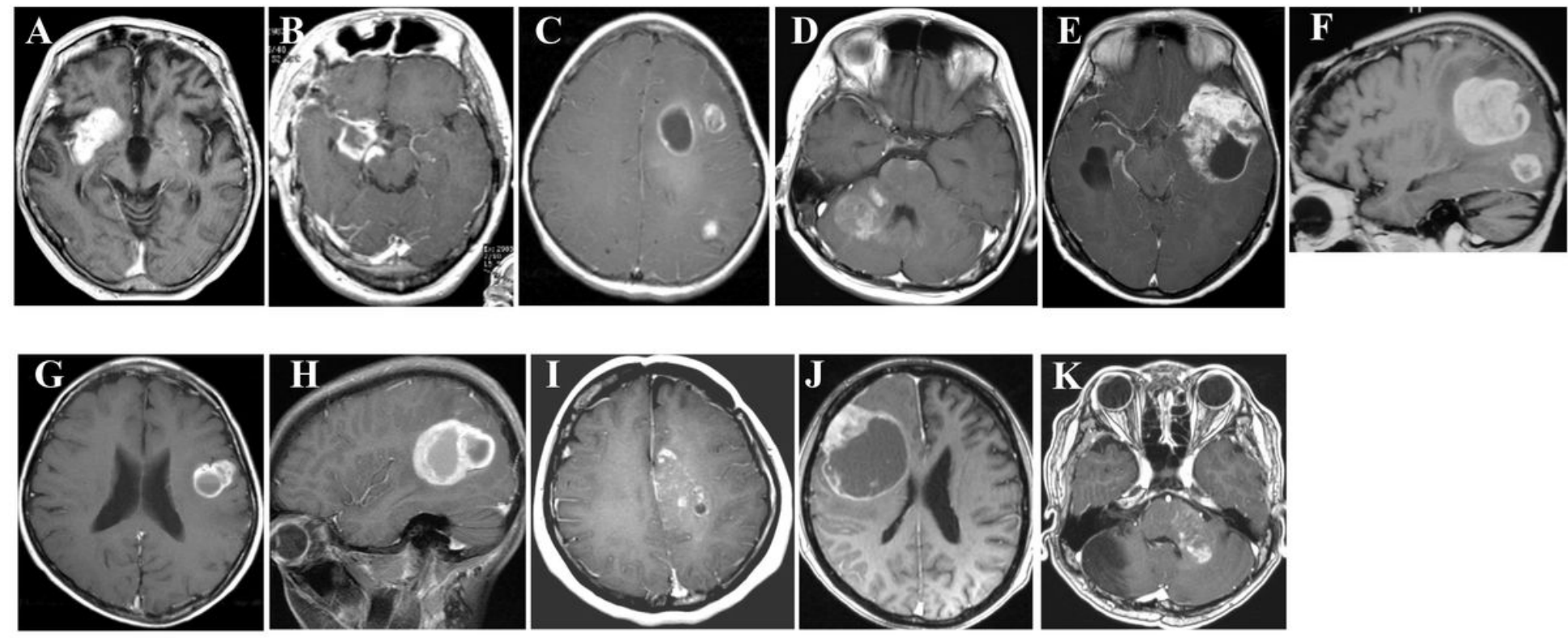

\section{Figure 1}

Representative preoperative gadolinium contrast-enhanced T1-weighted magnetic resonance images of radiation-induced gliomas. Images A-K correspond to Cases 1-11, respectively. 


\section{Figure 2}

A

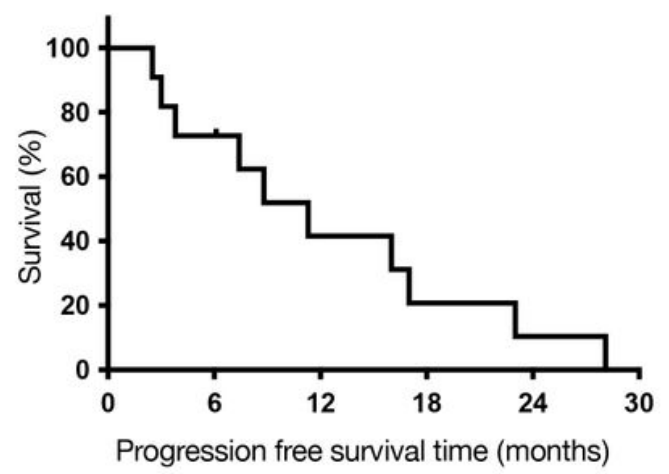

C

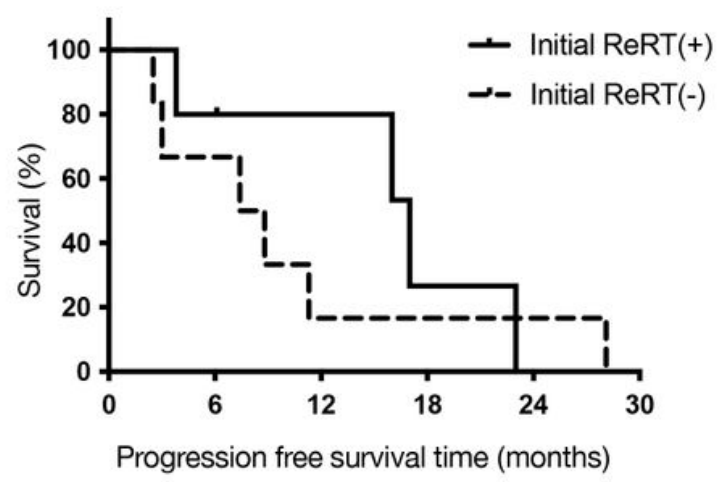

B

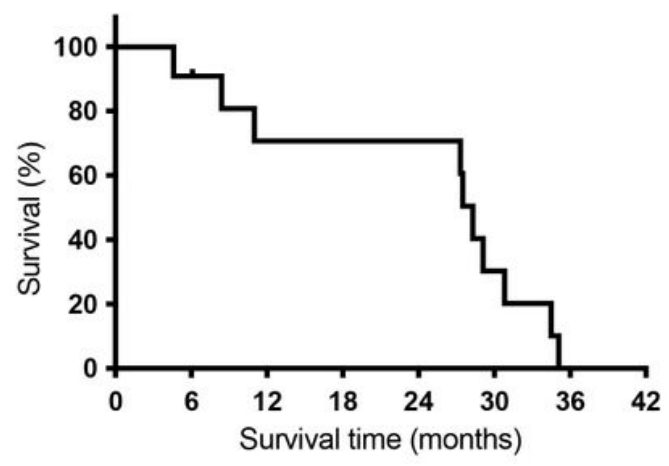

D

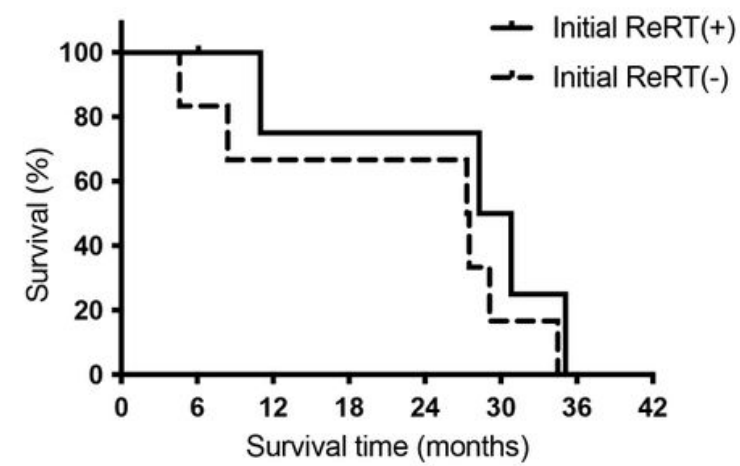

Figure 2

Kaplan-Meier curves of progression-free survival (A, C, E) and overall survival (B, D, F). (A) The median progression-free survival time was 10.1 months. (B) The median overall survival time was 27.5 months. (C) The median progression-free survival times were 16.5 months in patients treated with initial reirradiation ( $N=4)$ and 8.1 months in those treated without initial reirradiation $(N=6)(p=0.69)$. (D) The median overall survival times were 30.8 months in patients treated with initial reirradiation $(\mathrm{N}=4)$ and 27.4 months in those treated without initial reirradiation $(N=6)(p=0.21)$. 


\section{Figure 3}

B

A

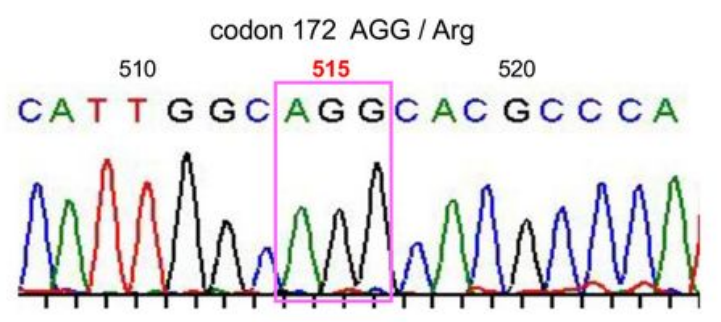

C

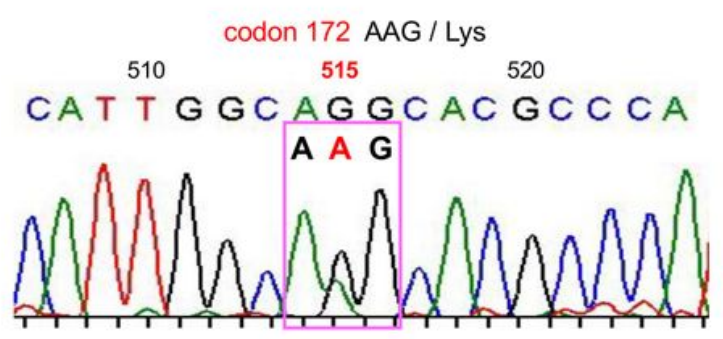

Figure 3

Magnetic resonance imaging and sequencing analysis of the IDH2 gene (A) T2-weighted magnetic resonance image at the initial presentation showing a hyperintense lesion in the left medial frontal lobe (black arrow). (B) Sequencing analysis of a glioblastoma showing the homozygous $\mathrm{G}$ at codon 515 in the IDH2 gene. (C) Sequencing analysis of a diffuse astrocytoma showing the heterozygous $G$ and $A$ at codon 515 in the IDH2 gene.

\section{Supplementary Files}

This is a list of supplementary files associated with this preprint. Click to download.

- 211106RIGTableJNO.xlsx 